\title{
Near Horizon Geometries and Black Hole Holograph
}

\author{
Jerzy Lewandowski ${ }^{1}$ * Istvan Racz ${ }^{2}$ 丹 and Adam Szereszewski团 \\ ${ }^{1}$ Faculty of Physics, University of Warsaw, \\ Pasteura 5, 02-093 Warsaw, Poland and \\ ${ }^{2}$ Wigner RCP, Konkoly Thege Miklós út 29-33, H-1121 Budapest, Hungary
}

(Dated: January 9, 2017)

\begin{abstract}
Two quasi local approaches to black holes are combined: Near Horizon Geometries (NHG) and stationary Black Hole Holographs (BHH). Necessary and sufficient conditions on BHH data for the emergence of NHGs as resulting vacuum solutions to Einstein's equations are found.
\end{abstract}

\section{Introduction}

In this letter we combine results of two topics of the quasi local theory of black holes $(\mathrm{BH})$. The first one is the theory of near horizon geometries (NHG) of extremal BHs $[1-3]$. They are exact solutions to Einstein's equations obtained by a naturally defined limit of neighborhoods of extremal (degenerate) Killing horizons. The first examples were derived from the extremal Reissner-Nordström solution and from the extremal Kerr. A larger family of examples (defined modulo an equation, that has to be solved, though) is set by the Kundt's class of solutions to Einstein's equations [4 10]. The second topic is the recent stationary Black Hole holograph (BHH) [11, 12]. This approach relies on the characteristic Cauchy problem for the electrovacuum Einstein's equations. If the transversal to each other null surfaces are non-expanding, then they become components of a bifurcated Killing horizon. The motivation for the current paper is an observation, that the NHGs also admit bifurcated Killing horizons. That makes them a special case of the BHHs. In the current letter we present a solution to the inverse problem, namely, we find conditions on the BHH data that are necessary and sufficient for the corresponding hologram spacetime to be a NHG. Our result may be considered as the first step in using the BHH construction in a quest for an interesting generalization of the idea of NHG. For simplicity, we will restrict here to $4 \mathrm{~d}$ spacetimes and the vacuum Einstein's equations.

A BHH data $(S, g, \omega)$ is: a compact 2-manifold $S$ (a BHH space) endowed with a metric tensor (a BHH metric tensor)

$$
g=g_{A B} d x^{A} d x^{B}
$$

\footnotetext{
*Jerzy.Lewandowski@fuw.edu.pl + racz.istvan@wigner.mta.hu † Adam.Szereszewski@fuw.edu.pl
} 
and a 1-form (a BHH 1-form)

$$
\omega=\omega_{A} d x^{A}
$$

where $\left(x^{A}\right)=\left(x^{1}, x^{2}\right)$ is a local coordinate system at $S$. Note that this is a geometric version of the original definition [11, 12]. The corresponding hologram is a $4 \mathrm{~d}$ spacetime in which the 2-space $S$ becomes the intersection between two non-expanding null surfaces (Non-Expanding Horizons [13 15]), while $g$ becomes the metric tensor induced in $S$. The 1-form $\omega$ becomes the pullback to $S$ of the rotation 1-form potential of one of the horizons, and, respectively, minus the rotation 1-form potential of the other one pulled back to $S$. The spacetime geometry is determined via the characteristic Cauchy problem for vacuum Einstein's equations in the causal future and in the past of the intersection $S$. The BHH theorem states that in this spacetime the non-expanding horizons set a bifurcated Killing horizon. Among all the black hole spacetimes obtained in that way there are also all the NHGs. Indeed, it is known, that each NHG contains a bifurcated Killing horizon [4, 16]. We will find below necessary and sufficient conditions on $(S, g, \omega)$ for the corresponding hologram to be a NHG.

\section{The Black Hole Holograph}

Given a BHH data $(S, g, \omega)$ the hologram spacetime manifold $M$ has the product topology

$$
M \sim S \times \mathbb{R} \times \mathbb{R}
$$

The coordinates $\left(x^{A}\right)$ defined on $S$, as well as coordinates $u$ and $v$ defined on the first, and the second factor $\mathbb{R}$, respectively, are naturally extended to the Cartesian product. The surfaces

$$
N_{1} \text { such that } u=0 \text { and } N_{2} \text { such that } v=0 \text {, }
$$

respectively, are assumed to be null and non-expanding with respect to the resulting spacetime geometry, while $S$ is identified with the surface $u=v=0$ in $M$. According to the standard characteristic Cauchy problem for vacuum Einstein's equations, in the smooth case, the spacetime geometry is determined (up to remaining diffeomeorphisms) in the wedges $u \geq 0, v \geq 0$ and $u \leq 0, v \leq 0$, in some neighborhood of $S=N_{1} \cap N_{2}$, provided that the following conditions hold at the surfaces $N_{1}$ and $N_{2}$, and at $S$, respectively:

- The pullback of the spacetime metric to each of the surfaces $N_{1}$, and $N_{2}$ respectively, is the following degenerate metric

$$
g_{A B} d x^{A} d x^{B}
$$


- The vectors $\ell=\partial_{u}, n=\partial_{v}$ are future oriented and satisfy

$$
\left.\nabla_{\ell} \ell\right|_{N_{1}}=0,\left.\quad \nabla_{n} n\right|_{N_{2}}=0 .
$$

- The pull back to $S$ of the 1 -form $-n_{\mu} \nabla_{\nu} \ell^{\mu}$ is

$$
-\left.n_{\mu} \nabla_{A} \ell^{\mu}\right|_{S}=\omega_{A}
$$

The BHH theorem [11, 12] states, that the spacetime metric tensor determined by the data admits a Killing vector $K$, that, using the remaining diffeomorphisms, can be given the form

$$
K=u \partial_{u}-v \partial_{v} .
$$

Therefore, the surfaces $N_{1}$ and $N_{2}$ form a (non-extremal) bifurcated Killing horizon while the pullback of the 1-form $\omega_{\nu}=-n_{\mu} \nabla_{\nu} \ell^{\mu}$ to $N_{2}$ is its rotation 1-form potential. In this sense the construction works as a stationary BH holograph: given any 2-dimensional data $(S, g, \omega)$ it produces 4-dimensional spacetime in the domain of dependence of the bifurcate Killing horizon, $N_{1} \cup N_{2}$.

\section{3. $N H G$ from $B H H$}

Suppose now, that a $\mathrm{BHH}$ data $(S, g, \omega)$ satisfies the following equation

$$
\omega_{(A ; B)}+\omega_{A} \omega_{B}-\frac{1}{2} R_{A B}=0
$$

where by ';' we denote the torsion free covariant derivative defined on $S$ by the metric $g$, and $R_{A B}$ is the Ricci tensor of $g$. This equation is soluble [4, 17, 18] only when $S$ is either a topological 2-sphere

$$
S=S_{2}
$$

or a 2-torus

$$
S=S_{1} \times S_{1}
$$

In the latter case, the only solution is

$$
\omega_{A}=0=R_{A B},
$$

therefore we will be assuming henceforth, that the manifold $S$ is a 2 -sphere $S_{2}$. Whenever (9) holds, the hologram metric tensor can be written down explicitly. Indeed, the following metric tensor

$$
d s^{2}=-2 d u\left(d v-2 v \omega-\frac{1}{2} v^{2}\left[\omega_{A ;}{ }^{A}+2 \omega_{A} \omega^{A}\right] d u\right)+g_{A B} d x^{A} d x^{B}
$$


is an exact solution of the vacuum Einstein equations [4] that matches the hologram data (5)-(7). Owing to uniqueness (mod diffeomorphisms) in BHH [12], this is the corresponding $\mathrm{BH}$ hologram. Such geometries are called Near Horizon. A remarkable property of this $\mathrm{BH}$ hologram (10) is emergence of a second Killing vector field, namely

$$
L=\partial_{u}
$$

The surface $N_{2}$ is extremal Killing horizon of the Killing vector field $L$, still being a component of the bifurcated non-extremal horizon of the Killing vector field $K$. Therefore, our first conclusion is, that every BHH data $(S, g, \omega)$ such that the equation (9) is satisfied, defines a NHG with the extremal Killing horizon $N_{2}$.

\section{Flipped $N H G$ BHH data}

The gauge freedom we have in setting up the initial data for $\mathrm{BHH}$ [12] yields some ambiguity in identifying NHGs. For instance, condition (9) is not necessary as other BHH data may also define a NHG as the hologram spacetime. For example, the following transformation in the space of the holographic data

$$
(S, g, \omega) \mapsto(S, g,-\omega)
$$

corresponds to switching of the factors in $S \times \mathbb{R} \times \mathbb{R}$, namely

$$
\left(x^{A}, u, v\right) \mapsto\left(x^{A}, v, u\right),
$$

because on $S=N_{1} \cap N_{2}$

$$
-\left(\partial_{v}\right)_{\mu} \nabla_{A}\left(\partial_{u}\right)^{\mu}=\left(\partial_{u}\right)_{\mu} \nabla_{A}\left(\partial_{v}\right)^{\mu}
$$

holds. Hence, every data $(S, g, \omega)$ which satisfies the switched equation (9), that is

$$
\omega_{(A ; B)}-\omega_{A} \omega_{B}+\frac{1}{2} R_{A B}=0
$$

also defines a NHG, this time with the extremal horizon $N_{1}$.

\section{A general case of $\mathrm{NHG}$ from $\mathrm{BHH}$}

An analogous gauge freedom explains that the most general form of condition (9) upon which $\mathrm{BHH}$ data still gives rise to NHG such that $N_{2}$ becomes the extremal Killing horizon comes with the following gauge transformation

$$
(S, g, \omega) \mapsto(S, g, \omega+d \lambda)
$$


where $\lambda: S \rightarrow \mathbb{R}$ is an arbitrary function (differentiable suitable number of times). It may be obtained by the following coordinate transformation

$$
\left(x^{A}, u, v\right) \mapsto\left(x^{A}, e^{-\lambda} u, e^{\lambda} v\right) .
$$

It follows that every data $(S, g, \omega)$ such that there is a function $\lambda: S \rightarrow S$ such that

$$
\omega_{(A ; B)}+\lambda_{; A B}+\left(\omega_{A}+\lambda_{, A}\right)\left(\omega_{B}+\lambda_{, B}\right)-\frac{1}{2} R_{A B}=0
$$

also defines a NHG. This non-linear equation on the unknown function $\lambda$ can be written as a linear equation on a new nowhere vanishing function

$$
f:=e^{\lambda} \text {. }
$$

Indeed, that substitution turns the equation (17) into (below, ' $D^{\prime} \equiv{ }^{\prime} ;{ }^{\prime}$ )

$$
\left(D_{A} D_{B}+\omega_{A} D_{B}+\omega_{B} D_{A}+\omega_{(A ; B)}+\omega_{A} \omega_{B}-\frac{1}{2} R_{A B}\right) f=0 .
$$

6. Necessary and sufficient condition on BHH to give rise to $N H G$ with respect to $\mathrm{N}_{2}$.

It turns out, that not every BHH data $\left(S_{2}, g, \omega\right)$ admits a solution $f$ to (18). The solubility conditions are expressed by the following BHH data invariants [13, 19]:

(i) the Ricci scalar

$$
R=R_{A B} g^{A B}
$$

of the $2 \mathrm{~d}$ metric tensor $g$, and

(ii) the rotation invariant

$$
\star d \omega
$$

and its scalar potential $U$, defined by the following equation

$$
\Delta U=\star d \omega,
$$

where $\Delta$ and $\star$ are the Laplace operator and Hodge star respectively defined on $S_{2}$ by the metric $g$.

The necessary solubility conditions are

1. The complex valued function $R+2 i \Delta U$ nowhere vanishes, i.e.

$$
R+2 i \Delta U \neq 0 \text { for every } x \in S_{2} .
$$


2.

$$
\frac{R+2 i \Delta U}{R-2 i \Delta U} e^{6 i U}=\text { const }
$$

3. The function

$$
\tilde{f}:=(R-2 i \Delta U)^{-\frac{1}{3}} e^{i U}
$$

and the 1-form

$$
\tilde{\omega}:=\star d U \text {. }
$$

satisfy equation (18), that is

$$
\left(D_{A} D_{B}+\tilde{\omega}_{A} D_{B}+\tilde{\omega}_{B} D_{A}+\tilde{\omega}_{(A ; B)}+\tilde{\omega}_{A} \tilde{\omega}_{B}-\frac{1}{2} R_{A B}\right) \tilde{f}=0 .
$$

The meaning of Condition 2 is, that the function $(R+2 i \Delta U) e^{3 i U}$ is real valued up to a constant factor. The factor can be absorbed into the potential $U$.

Notably Conditions 1-3 are also sufficient for the existence of a nowhere vanishing function $f$ satisfying equation (18). To see this note that such an $f$ can be given as

$$
f:=B \tilde{f},
$$

where $\tilde{f}$ is a solution to (23), whereas the function $B$ is given as

$$
\Delta \ln B:=\star d \star \omega .
$$

\section{Non-rotating BHH data}

An example of a $\mathrm{BHH}$ data $\left(S_{2}, g, \omega\right)$, such that our Conditions 1-3 are not satisfied is a non-rotating case when

$$
d \omega=0 \text {. }
$$

In this case the equations (23)-(25) imply [13, 19]

$$
0=\int_{S_{2}} d^{2} x \sqrt{\operatorname{det} g}\left(\Delta-\frac{1}{2} R\right) R^{-\frac{1}{3}}=-\frac{1}{2} \int_{S_{2}} d^{2} x \sqrt{\operatorname{det} g}\left(R^{\frac{1}{3}}\right)^{2},
$$

a condition requiring the vanishing of $R$ throughout $S_{2}$. This, however, leads to a contradiction as a 2-sphere does not admit a flat metric tensor which, in turn, verifies non-existence of non-rotating vacuum NHG [20]. 


\section{Summary and outlook}

The subset of BHH data that corresponds to NHGs was identified. It was shown that, up to the flips of the horizons, a BHH data $\left(S_{2}, g, \omega\right)$ gives rise to a NHG if and only if Conditions 1-3 hold. A general exact solution to the involved constraints is not known. The found relation between the BHH construction and the NHGs may play significant role in attempting to give suitable generalizations of the concept of NHG. The NHGs are exact solutions to Einstein's equations that at the same time provide the 0th order in a suitable expansion of spacetime metric about an extremal Killing horizon. In the non-extremal case a suitable generalization of the NHGs is not known yet.

\section{Acknowledgements}

This work was partially supported by the Polish National Science Centre grant No. 2015/17/B/ST2/02871 and by the Hungarian NKFIH grant K-115434.

[1] H. K. Kunduri, J. Lucietti, Classification of Near-Horizon Geometries of Extremal Black Holes, Living Rev. Rel. 16 (2013), 8, http://www.livingreviews.org/lrr-2013-8, arXiv:abs/1306.2517.

[2] H. S. Reall, Higher dimensional black holes and supersymmetry, Phys. Rev. D 68 (2003), 024024, arXiv:hep-th/0211290.

[3] J. M. Bardeen, G. T. Horowitz, The Extreme Kerr throat geometry: A Vacuum analog of $A d S(2) \times S^{2}$, Phys. Rev. D 60 (1999), 104030, arXiv:hep-th/9905099.

[4] T. Pawłowski, J. Lewandowski, J. Jezierski, Spacetimes foliated by Killing horizons, Class. Quantum Grav. 21 (2004), 1237-1252, arXiv:gr-qc/0306107.

[5] J. Podolský, M. Žofka, General Kundt spacetimes in higher dimensions, Class. Quantum Grav. 26 (2009), 105008.

[6] P. Krtouš, J. Podolský, A. Zelnikov, H. Kadlecová, Higher-dimensional Kundt waves and gyratons, Phys. Rev. D 86, 044039.

[7] J. Podolský, R. Švarc, Physical interpretation of Kundt spacetimes using geodesic deviation, Class. Quantum Grav. 30 (2013), 205016.

[8] J. Lewandowski, A. Szereszewski, P. Waluk, When Isolated Horizons met Near Horizon Geometries, 2nd LeCosPA Symposium Proceedings, "Everything about Gravity" celebrating the centenary of Einstein's General Relativity, December 14-18 (2015), Taipei.

[9] J. Lewandowski, A. Szereszewski, P. Waluk, Spacetimes foliated by non-expanding and Killing horizons: higher dimension, Phys. Rev. D 94 (2016), 064018.

[10] H. Stephani, D. Kramer, M. MacCallum, C. Hoenselaers, E. Herlt, Exact Solutions to Einstein's Field Equations, Cambridge University Press, 2003.

[11] I. Rácz Stationary black holes as holographs, Class. Quantum Grav. 24 (2007), 5541-5571.

[12] I. Rácz Stationary black holes as holographs II., Class. Quantum Grav. 31 (2014), 035006.

[13] A. Ashtekar, C. Beetle, J. Lewandowski, Geometry of Generic Isolated Horizon, Class. Quantum Grav. 19 (2002), 1195-1225, arXiv:gr-qc/0111067. 
[14] A. Ashtekar, B. Krishnan, Isolated and Dynamical Horizons and Their Applications, Living Rev. Rel. 7 (2004), 10, http://www.livingreviews.org/lrr-2004-10, arXiv:gr-qc/0407042.

[15] J. Lewandowski, T. Pawłowski, Quasi-local rotating black holes in higher dimension: geometry, Class. Quantum Grav. 22 (2005), 1573-1598, arXiv:gr-qc/0410146.

[16] I. Rácz and R. M. Wald Extension of spacetimes with Killing horizon, Class. Quantum Grav. 9 (1992), 2643-2656.

[17] J. Jezierski, On the existence of Kundt's metrics and degenerate (or extremal) Killing horizons, Class. Quantum Grav. 26 (2009), 035011, arXiv:0806.0518 [gr-qc].

[18] J. Jezierski, B. Kaminski, Towards uniqueness of degenerate axially symmetric Killing horizon, Gen. Relativ. Gravit. 45 (2016), 987-1004, arXiv:1206.5136 [gr-qc].

[19] J. Lewandowski, T. Pawłowski, Extremal Isolated Horizons: A Local Uniqueness Theorem, Class. Quantum Grav. 20 (2003), 587-606, arXiv:gr-qc/0208032.

[20] P. T. Chrusciel, H. S. Reall, P. Tod, On non-existence of static vacuum black holes with degenerate components of the event horizon, Class. Quantum Grav. 23 (2006), 549-554. 